\title{
Editorial to the theme issue on model-driven service engineering
}

\author{
Juan Manuel Vara • Mike Papazoglou • Il-Yeol Song
}

Received: 8 July 2013 / Accepted: 14 July 2013 / Published online: 25 July 2013

(C) Springer-Verlag Berlin Heidelberg 2013

\section{Introduction}

During the last years, Model-Driven Engineering (MDE) has started to have a direct influence in other research fields. Under the light of the premise that everything is a model, coined by Jean Bézivin in 2004 and adopted by the MDE community since then, practitioners from other areas have discovered that they were able to express their problems in terms of models and then take advantage of MDE techniques to solve them-or at least simplify them, either by increasing the level of automation or by raising the abstraction level at which solutions are planned and developed. The scope varies widely, from generic domains, like Web Engineering (see the previous SoSyM Theme Issue on Model-Driven Web Engineering), to more specific ones, like DB schema matching or domotics.

Out of doubt, one of the areas that has more decisively benefited from MDE advances has been Service Engineering. It aims at bringing together the benefits of Service Orientation and Business Process Management, therefore making the most of Service Orientation to help organizations deliver sustainable business value with increased agility and cost effectiveness. To that end, services are widely used as software systems, and the focus is shifted to business process as

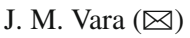

Universidad Rey Juan Carlos, Madrid, Spain

e-mail: juanmanuel.vara@urjc.es

M. Papazoglou

Tilburg University, Tilburg, The Netherlands

e-mail: m.p.papazoglou@ tilburguniversity.edu

I.-Y. Song

Drexel University, Philadelphia, PA, USA

e-mail: song@drexel.edu
}

key and determinant elements for the design of Information Systems.

In this context, MDE tools and techniques have largely served to foster the adoption and deployment of servicebased proposals. In fact, the use of models and modeling tools fits perfectly with some of the most important challenges that the development of service-based systems entails. For instance, model transformations have proven to be useful to provide a smooth transition between system specifications defined at different abstraction levels and thus to cope with the inherent complexity of aligning high level business specifications with low-level Service-Oriented Architectures (SOA).

As a result, several works on the provision of model-based support for service-based proposals have been developed during the last few years, giving rise to a new research area commonly referred as Model-Driven Service Engineering. The MoSE (Model-Driven Service Engineering) Workshop series, namely MoSE 2009 held at the CKIM conference in Honk-Kong (China) and MoSE 2010, held at the TOOLS conference in Malaga (Spain) served as a forum to publish the first results in the field.

This theme issue was conceived with the intention of providing a general overview of the latest advances in the field, with the belief that the certain levels of maturity gained by MDE a couple of years later had settled a solid basis to boost the development and improvement of service-based proposals.

\section{In this issue}

The call for papers for this theme issue was delivered by the end of 2011. As a response, 30 abstracts from 15 different countries were received and 22 out of them became 
full submissions. These submissions underwent a rigorous review process performed by an international group of the most recognized experts in the areas of MDE and Service Engineering. Their recommendations did not only serve to drive the selection of papers but also to polish the 6 papers that were finally recommended for publication. The result is a set of high-quality works that illustrate the advantages brought by the synergy established between Model-Driven Engineering and Service Engineering in different scenarios.

Opposite to most of the research in the area, mostly oriented to the application of MDE principles to service-based proposals, the first work of this theme issue goes the other way round, showing that MDE can also benefit from the principles of Service Orientation. In his paper "On the Modeling and Generation of Service-Oriented Tool Chains", Matthias Biehl, Jad El-Khoury, Frédéric Loiret and Martin Törngren applied the principles of SOA to solve the challenges of tool integration.

In "Model-Driven Engineering of Middleware-based Ubiquitous Services" Marco Autili, Mauro Caporuscio, Valérie Issarny and Luca Berardinelli put MDE principles to work to provide tool support for ubiSOAP, a ServiceOriented Middleware for ubiquitous networking. The result is an MDE environment to develop ubiquitous applications that constitutes a clear example of one the main benefits of MDE: the provision of effective techniques for the (semi-)automatic generation of service-oriented systems.

Next, two of the papers included in this theme issue deal with the challenge of developing secure servicebased systems. In "Modeling and Enforcing Secure Object Flows in Process-driven SOAs: An Integrated Model-driven Approach", Bernhard Hoisl, Stefan Sobernig and Mark Strembeck lean on MDE tools and techniques to provide an Eclipse-based solution to ensure the confidentiality and the integrity of object flows in process-driven systems. On the other hand, Mukhtiar Memon, Gordhan D. Menghwar, Mansoor H. Depar and Akhtar A. Jalbani present a proposal to include the modeling of security concerns in SOAs from the early stages of development in "Security Modeling for Service-oriented Systems using Security Pattern Refinement Approach."
Probably, the best example of the synergy between service orientation and MDE is the level of adoption of Business Process Modeling (BPM) as a common practice in the specification and development of service-based systems. A well-known issue of BPM is the lack of support to consider non-functional requirements and QoS. To alleviate this problem, Paolo Bocciarelli and Andrea D'Ambrogio introduce a BPMN extension for the specification of performance and reliability properties in "A Model-driven method for enacting the design-time QoS analysis of Business Processes"

Finally, the use of orchestrators to coordinate the execution of the services inherent to service composition results in single point failure and communication overhead scenarios that should be avoided. A possible workaround is the adoption of (semi-)decentralized approaches like the one presented in "Heuristics for Constrained Composite Web Service Decentralization" by Walid Fdhila, Marlon Dumas, Claude Godart and Luciano García-Bañuelos. The authors go beyond their previous proposal, based on manual grouping of activities into partitions, by defining a formal method which considers a number of variables to drive the partition process. Such method poses a quadratic optimization problem which the authors address by combining a greedy algorithm and a Tabu search.

All in all, the papers presented in this theme issue provide a representative sample of the possibilities opened by the combination of models and modeling languages and service orientation. We hope you will enjoy reading this theme issue and will find these works inspiring.

Acknowledgments First of all, we would like to thank the authors for their work and patience since the number of submissions resulted in a long reviewing process. Such process would never have been completed without the hard and extremely thorough work of the reviewers to whom we are deeply grateful. Besides, we would like to express our appreciation to the Editors-in-Chief for hosting this theme issue and particularly to Martin Schindler for his continuous support and his constant guidance along the preparation process. Finally, this theme issue has been partially supported by the MASAI project, financed by the Spanish Ministry of Science and Technology (Ref. TIN2011-22617). 